\title{
Reinis, Austra, Reforming the Art of Dying. The ars moriendi in the German Reformation (1519-1528)
}

Jean-Luc Le Cam

\section{OpenEdition}

\section{Journals}

Édition électronique

URL : http://journals.openedition.org/ifha/2214

DOI : 10.4000/ifha.2214

ISSN : 2198-8943

Éditeur

IFRA - Institut franco-allemand (sciences historiques et sociales)

Référence électronique

Jean-Luc Le Cam, «Reinis, Austra, Reforming the Art of Dying. The ars moriendi in the German

Reformation (1519-1528) », Revue de l'IFHA [En ligne], Date de recension, mis en ligne le 01 janvier 2010, consulté le 22 septembre 2020. URL : http://journals.openedition.org/ifha/2214 ; DOI : https://doi.org/ 10.4000/ifha.2214

Ce document a été généré automatiquement le 22 septembre 2020.

(C)IFHA 


\title{
Reinis, Austra, Reforming the Art of Dying. The ars moriendi in the German Reformation (1519-1528)
}

\author{
Jean-Luc Le Cam
}

1 Luther constatait : «Beaucoup de livres ont été écrits pour nous dire comment nous devons nous préparer à la mort, mais on n'y trouve que des erreurs et les gens en ont seulement été encore plus troublés » (Luthers Werke, WA 41, p. 699). Sa critique portait donc à la fois sur le contenu théologique de ces livres et sur le ressenti des fidèles, sur l'intelligence et l'émotion. Ce sont les deux axes que choisit d'emprunter cet ouvrage, issu d'une thèse de doctorat en histoire de la Réforme à la faculté de théologie de Princeton, pour étudier 16 ouvrages de réformateurs traitant de l'art de mourir entre 1519 et 1528. Il s'agit selon A.R. de retracer comment Luther et 13 autres auteurs ont cherché, dans cette courte période d'une décennie, à " réformer l'art de mourir » en fonction des nouvelles orientations théologiques mais aussi de nouveaux procédés rhétoriques.

2 Les sources mobilisées dans ce travail sont donc uniquement ces livres désignés, quels que soient leur titre, sous l'appellation générique de Sterbebücher. Ils appartiennent à un genre littéraire particulier, que l'auteure, plutôt que de parler de littérature de dévotion, classe à la suite de Bernd Hamm dans la catégorie de la «théologie de la piété » (Frömmigkeitstheologie), destinée à transcrire dans la vie courante les enseignements religieux. Cette théologie s'adresse non pas aux doctes mais aux simples prêtres ou membres de communauté monastique ainsi qu'aux laïcs. Luther a pris lui-même une vaste part à ce genre littéraire qui l'a plus fait connaître en son temps que ses grands textes réformateurs (ainsi ses écrits sur le baptême, le mariage, l'eucharistie, la confession, l'extrême-onction). À cause de la qualité de leurs auteurs et du niveau des tirages, ces ouvrages touchaient une très large audience, si bien que leur étude contribue aussi à la connaissance de la dissémination de la Réforme. Par ailleurs, l'auteure plaide, à la suite des derniers travaux sur ces sujets, pour une approche non seulement théologique de cette production mais qui fasse droit aussi à la dimension 
affective et émotionnelle de la piété. Martin Jung, Virgile Stoltz ont par exemple insisté sur la part des sentiments dans l'expérience réformatrice de Luther et montré que sa rhétorique puisait sa force dans son anthropologie, selon laquelle le cœur est le lieu à la fois de la compréhension intellectuelle, de l'émotion et de la volonté. C'est pourquoi le prêche devait s'adresser à la fois à l'intellect et à l'émotion.

3 Ces 16 " pamphlets » en allemand sur la préparation à la mort sont chronologiquement compris entre 1519, date du sermon de Luther Von der bereytung zum Sterben, qu'on peut considérer comme le premier ars moriendi de la Réforme, et 1529, année de la publication de son Catéchisme. L'auteure ne fournit malheureusement aucune justification satisfaisante du choix de cette dernière borne chronologique, si ce n'est que « cette période est considérée généralement comme la période pré-confessionnelle ». Ces textes ont en commun de diffuser le message central de la Réforme sur la justification par la foi et la certitude du salut, ils transmettent aussi une nouvelle compréhension de la tentation qui menace le chrétien sur son lit de mort, ainsi que des sacrements et du sens de la Passion.

Le premier chapitre (2, car l'introduction est numérotée) fait à titre de comparaison le point sur le contenu des arts de mourir de la fin du Moyen Âge. La doctrine médiévale de l'incertitude du salut, combinée avec l'enseignement que l'homme peut, par sa propre volonté, faire ce qu'il faut pour mériter celui-ci, est alors le fondement de la consolation offerte au mourant.

5 Puis, l'auteure répartit les 16 textes de son corpus en trois catégories, selon des critères formels qui servent au découpage de l'ouvrage. La première est celle des sermons proprement dits, qui traitent de sujets à méditer soit en anticipation à la mort, soit directement sur son lit de mort. Un chapitre entier (3) est consacré à la pierre angulaire de cette nouvelle littérature qu'est le sermon de Luther de 1519. L'auteur y accorde une attention particulière à l'emploi de la rhétorique comme instrument émotionnel et intellectuel de la consolation. Rejetant l'idée traditionnelle que l'homme doit et peut contribuer à son salut, Luther cherche à enseigner que les trois sacrements sur le lit de mort, que sont la confession, l'Eucharistie et l'extrême onction, signifient que la victoire du Christ sur le mal, le péché et l'enfer, est aussi la victoire du chrétien. Il exhorte donc le chrétien mourant à recevoir les sacrements dans la joie avec la confiance que sa propre mort, son péché et l'enfer ont été vaincus par la Passion du Christ et que son salut est assuré. Le contexte historique, le contenu et la structure, les caractéristiques théologiques et rhétoriques du texte sont ensuite déroulés avec un excursus sur la théologie sacramentelle de Luther développée entre ce sermon et le traité De la captivité babylonienne de l'Église.

6 Le chapitre 4 passe ensuite en revue sept autres sermons sur la préparation à la mort, d'auteurs bien connus tels Johann Oecolampade, Georg Spalatin, et Johann Bugenhagen, ou plus obscurs. L'analyse se fait sur le même mode que dans le chapitre précédent, malheureusement pour chacun des auteurs (avec toutefois une insistance sur le Nunc dimittis d'Oecolampade - 1521), ce qui conduit à une accumulation assez fastidieuse et monotone d'analyses textuelles. Quelques observations en conclusion permettent toutefois de ramasser certaines caractéristiques générales. Au-delà des éléments déjà présents dans le sermon de Luther, on relève quelques variations entre ces auteurs, par exemple sur la mention ou non de la réception des sacrements sur le lit de mort, attestant une certaine liberté du genre par rapport à son modèle. 
7 La deuxième catégorie (chap. 4) regroupe les manuels à l'usage du lit de mort, que ce soit pour les mourants eux-mêmes ou pour ceux qui les assistent, et qui comprennent pour cette raison parfois des éléments de liturgie. Seul Oecolampade, qui présente d'ailleurs l'unique manuel liturgique adressé explicitement à l'usage du clergé, est un auteur connu parmi les cinq exemplaires de cette catégorie. L'auteure exploite plus particulièrement l'Evangelische lere d'un anonyme, parce qu'elle traite du problème de la mort d'un luthérien dans un environnement non luthérien, en insistant particulièrement sur la notion d'Anfechtung (attaque, tentation).

8 Le chapitre 6 est consacré aux instructions sur la mort comprises dans les sommaires ou résumés de la doctrine de la Réforme, qui n'appartiennent donc pas strictement au genre des Sterbebücher, mais peuvent jouer ce rôle par une partie de leur contenu. Selon Bernd Möller et Karl Stackamnn, ils se sont multipliés dans cette période troublée pour permettre aux chrétiens d'y voir plus clair en distinguant entre l'essentiel et l'accessoire. Les quatre ouvrages relevant de cette typologie, d'auteurs peu connus, portent des titres variés qui ne rendent pas forcément compte de leur nature.

9 La conclusion générale reprend sans grande ouverture les thèmes déjà ressassés. Ces premiers arts de mourir réformés s'appuient sur le sentiment que leurs lecteurs potentiels désespéraient pour la plupart de leur salut. Ils cherchaient à les convaincre que la victoire du Christ en croix le leur assurait. Ceci constituait une rupture radicale avec la doctrine traditionnelle qui voulait que personne ne puisse présumer avec certitude de son destin après la mort. L'analyse rhétorique de ces textes rejoint les recherches récentes sur l'histoire des émotions en s'efforçant de comprendre la dimension affective de l'enseignement des réformateurs sur la mort. Ayant eux-mêmes trouvé paix et joie dans la certitude du salut, les premiers auteurs d'arts de mourir réformés faisaient tout leur possible pour persuader les futurs mourants qu'eux aussi pouvaient faire face à la mort avec joie et confiance.

10 L'ouvrage, au total, déçoit par son manque de perspectives et d'interactions avec les différents secteurs de l'histoire culturelle, religieuse et sociale de la Réforme. Le caractère très scolaire de la construction, qui consiste à examiner l'un après l'autre, en suivant un plan quasiment identique, les textes qui servent de source à cette étude, y contribue certainement pour une part. Mais aussi la relative pauvreté de l'analyse textuelle qui se limite à repérer les contenus théologiques et les ressorts rhétoriques un par un, aux limites parfois de la simple paraphrase. Rien ne vient par ailleurs justifier le fait qu'on se limite à une période aussi courte. Il eût certainement été plus intéressant de poursuivre l'étude du genre pendant au moins un demi-siècle pour voir si l'institutionnalisation des Églises luthériennes avait eu des conséquences sur l'accompagnement des chrétiens vers la mort. Sur le sujet des transformations de la conception de la mort sous l'influence de Réforme luthérienne, on conseillera plutôt aux lecteurs anglophones l'analyse bien plus complexe et suggestive d'un autre chercheur américain : Craig M. Koslofsky, The Reformation of the Dead, Dead and Ritual Early Modern Germany, 1450-1700, Basingstoke/ New York : Palgrave, 2000 (voir compte-rendu dans : BullMHFA, 41, 2005, p. 435-437).

11 Jean-Luc Le Cam (Université de Bretagne Occidentale, Brest) 